\title{
Strategies to reduce intraluminal clot formation in endoscopically harvested saphenous veins
}

\author{
Emile N. Brown, BS, ${ }^{a}$ Zachary N. Kon, BA, ${ }^{\text {a }}$ Richard Tran, BS, ${ }^{a}$ Nicholas S. Burris, BS, ${ }^{a}$ Junyen Gu, MD, PhD, ${ }^{a}$ \\ Patrick Laird, BA, ${ }^{a}$ Philip S. Brazio, BS, BA, ${ }^{\text {a }}$ Seeta Kallam, MD, ${ }^{a}$ Kimberly Schwartz, RNFA, MBA, ${ }^{\text {a }}$ Lisa Bechtel, PA-C, ${ }^{\text {b }}$ \\ Ashish Joshi, MD, MPH, ${ }^{\mathrm{c}}$ Shaosong Zhang, MD, PhD, ${ }^{d}$ and Robert S. Poston, MD ${ }^{\mathrm{a}}$
}

From the Department of Surgery, Division of Cardiac Surgery, ${ }^{\text {a }}$ University of Maryland School of Medicine, Baltimore, Md; the St Joseph Medical Center, ${ }^{\mathrm{b}}$ Baltimore, Md; the University of Maryland Baltimore County, Department of Information Systems, ${ }^{\mathrm{c}}$ Baltimore, Md, and LightLab Imaging, Inc, ${ }^{\mathrm{d}}$ Westford, Mass.

Lisa Bechtel, PA-C, reports consulting fees from Datascope Corp. Shaosong Zhang, MD, is an employee of LightLab Imaging, Inc. Robert S. Poston, MD, is supported by grants from Guidant Corporation, American Heart Association (0435318N), an Intramural Grant at the University of Maryland, pilot grant from the Tobacco Restitution Fund at the University of Maryland, NHLBI (RO1 HL084080-01A1), and LightLab Imaging, Inc (supplies for the performance of OCT only).

Read at the Eighty-seventh Annual Meeting of The American Association for Thoracic Surgery, Washington, DC, May 5-9, 2007.

Received for publication May 10, 2007; revisions received July 10, 2007; accepted for publication July 24, 2007.

Address for reprints: Robert S. Poston, MD, Associate Professor of Surgery, University of Maryland School of Medicine, 22 S Greene St, Room N4W94, Baltimore, MD 21201 (Email: rposton@ smail.umaryland.edu).

J Thorac Cardiovasc Surg 2007;134:1259-65 $0022-5223 / \$ 32.00$

Copyright () 2007 by The American Association for Thoracic Surgery

doi:10.1016/j.jtcvs.2007.07.042
Objective: Residual clot strands within the excised saphenous vein are an increasingly recognized sequela of endoscopic vein harvest. We hypothesized that endoscopic visualization facilitated by sealed carbon dioxide insufflation causes stagnation of blood within the saphenous vein. In the absence of prior heparin administration, this stasis provokes clot formation.

Methods: Forty consecutive patients having coronary artery bypass grafting underwent endoscopic vein harvest using sealed (Guidant VasoView, $\mathrm{n}=30$; Guidant Corp, Minneapolis, Minn) or open (Datascope ClearGlide, $\mathrm{n}=10$; Datascope Corp, Montvale, NJ) carbon dioxide insufflation followed by ex vivo assessment of intraluminal saphenous vein clot via optical coherence tomography. In the sealed carbon dioxide insufflation groups, clot formation was compared with (preheparinized, $\mathrm{n}=20$ ) and without (control, $\mathrm{n}=10$ ) heparin administration before endoscopic vein harvest, either at a fixed dose or titrated to an activated clotting time greater than 300 seconds. Risk factors for clot formation were assessed.

Results: Residual saphenous vein clot was a universal finding in control veins (sealed carbon dioxide insufflation endoscopic vein harvest without preheparinization). At either dose used, heparin given before endoscopic vein harvest significantly decreased saphenous vein clot burden. A similar reduction in clot was observed when using open carbon dioxide insufflation endoscopic vein harvest without preheparinization. Intraoperative blood loss and blood product requirements were similar in all groups. Patient age and preoperative maximum amplitude of the thrombelastography tracing showed a linear correlation with saphenous vein clot volume.

Conclusion: By enabling the quantification of this issue as never before possible, optical coherence tomography screening revealed that intraluminal saphenous vein clot is frequently found after endoscopic vein harvest. Systemic heparinization before harvest or an open carbon dioxide endoscopic vein harvest system are benign changes in practice that can significantly lessen this complication.

$\mathrm{T}$ he saphenous vein graft (SVG) remains the most commonly used surgical conduit in coronary artery bypass grafting (CABG) (Society of Thoracic Surgeons National Cardiac Database). Endoscopic vein harvest (EVH) has been rapidly adopted and is now used in $80 \%$ of cardiac surgery centers owing to dramatic improvements in wound morbidity and cosmesis compared with traditional open methods. ${ }^{1-3}$ A recent meta-analysis of randomized clinical trials found no adverse effect of endoscopic harvest on SVG patency. ${ }^{4}$ However, the studies included in this analysis enrolled small numbers of patients and used variable management protocols and EVH systems. More recently, the PREVENT IV multicenter CABG trial assessed 12-month patency in more than 4000 SVGs and found 


$$
\begin{aligned}
& \text { Abbreviations and Acronyms } \\
& \begin{aligned}
\text { CABG } & =\text { coronary artery bypass grafting } \\
\text { EVH } & =\text { endoscopic vein harvest } \\
\text { OCT } & =\text { optical coherence tomography } \\
\text { SV } & =\text { saphenous vein } \\
\text { SVG } & =\text { saphenous vein graft } \\
\text { TEG } & =\text { thromboelastography }
\end{aligned}
\end{aligned}
$$

the use of EVH to be an independent predictor of graft failure as compared with open harvest (odds ratio $=1.35$, $P<.001)^{5}$

It is not clear how EVH might increase the risk of SVG loss. Prior studies, ${ }^{2,6}$ including one from our own group, ${ }^{7}$ have reported no significant differences in intimal trauma for veins harvested via endoscopic versus open techniques. However, the presence of retained clot within the saphenous vein (SV) lumen has been an increasingly recognized complication of EVH. ${ }^{8}$ An intravascular imaging modality known as optical coherence tomography (OCT) provides a novel means for intraoperative assessment of the entire tract of harvested SV and thereby allows for the quantification of intraluminal clot burden. ${ }^{9}$ We hypothesized that carbon dioxide $\left(\mathrm{CO}_{2}\right)$ insufflation within a closed tunnel around the $\mathrm{SV}$, used to facilitate endoscopic visualization, causes stagnation of blood that leads to clot strand formation. The purpose of this study was to test this hypothesis by comparing clot burden defined by OCT after two different modifications to our current practice: (1) with and without heparin anticoagulation given before EVH initiation and (2) with the use of two different EVH devices that do and do not allow blood flow to be maintained in the SV during harvest (ie, open versus sealed $\mathrm{CO}_{2}$ insufflation).

\section{Materials and Methods}

\section{Patient Enrollment and Data Management}

After Institutional Review Board approval (University of Maryland, Baltimore, protocol 25350), 40 consecutive subjects scheduled for CABG with an SVG were enrolled. The first 10 patients were treated according to the standard of care at our institution: EVH using a sealed and constant method of $\mathrm{CO}_{2}$ insufflation (VasoView6; Guidant Corp, Minneapolis, Minn) without prior anticoagulation (control). An additional 20 patients in whom the VasoView EVH system was used received systemic heparin anticoagulation before the initiation of $\mathrm{EVH}$, either titrated to an activated clotting time greater than 300 seconds $(\mathrm{n}=10)$ or as a single 5000-IU intravenous bolus $(\mathrm{n}=10)$. The last 10 subjects underwent SV harvest without prior heparinization using an "open $\mathrm{CO}_{2}$ " EVH system (ClearGlide; Datascope Corp, Montvale, $\mathrm{NJ}$ ), which allows venous blood flow to be maintained during harvest. Demographics, preoperative risk factors and medications, and intraoperative and postoperative data were prospectively imported into a relational database.

\section{Surgical Technique and SVG Handling}

All patients underwent median sternotomy, followed by concurrent left internal thoracic artery harvest and EVH. Using either VasoView or ClearGlide system, we initiated EVH by insertion of a conical dissection cannula to create a tunnel around the vein. For the VasoView device, a seal was created within the tunnel by inflating a trochar port balloon to less than $5 \mathrm{~mL}$ and then $\mathrm{CO}_{2}$ was insufflated to a pressure of 10 to $12 \mathrm{~mm} \mathrm{Hg}$. With the ClearGlide EVH system, $\mathrm{CO}_{2}$ insufflation was used to facilitate endoscopic visualization, but without a sealed tunnel (Figure 1). Division of branches was performed with bipolar electrocautery set at 20 watts. Proximal SV ligation was performed through a separate stab incision. After removal from the leg, the SV was flushed and stored in a Plasma-Lyte solution (Baxter International, Inc, Deerfield, Ill) containing heparin, glyceryl trinitrate, and verapamil. ${ }^{10}$ Given that all patients underwent off-pump CABG, heparin administration was titrated to an activated clotting time greater than 300 seconds and a heparin level greater than $2 \mathrm{IU} / \mathrm{mL}$ according to protamine titration assay (HMS system; Medtronic, Inc, Minneapolis, Minn). Aprotinin was given intraoperatively to selected highrisk patients according to a previously established protocol. ${ }^{11}$ Perioperative aspirin was used in all patients $(325 \mathrm{mg}$ by mouth daily). Shed mediastinal blood was collected intraoperatively and retransfused with a cell salvage device (Cobe BRAT 2; CobeCardiovascular, Arvada, Colo). Perioperative coagulation was monitored by thromboelastography (TEG 5000; Haemoscope, Niles, Ill) activated by kaolin.

\section{Measurement of Intraluminal Clot}

All SVs were imaged intraoperatively after EVH with an imaging probe (ImageWire; LightLab Imaging, Westford, Mass) introduced into the vein during gentle saline infusion. Residual clot was identified within the SV lumen via cross-sectional OCT images and distinguished by the characteristic radial signal attenuation produced by thrombi as previously described..$^{8,12}$ Clot distribution within the tract of vein was documented by externally marking the location of the imaging probe, visualized through the vessel wall by the rotating infrared light at the catheter tip. This information was used to calculate the portion of vein that contains clot relative to the total conduit length (ie, "clot fraction"). The volume of clot within each conduit was also calculated off-line with proprietary software (LightLab Imaging). These analyses were performed by a technician blinded to group assignment.

So that the diagnosis of intraluminal clot could be confirmed histologically, biopsy specimens were procured from discarded SV segments registered to the area of intraluminal clot visualized via OCT imaging. These "image-guided" biopsy specimens were embedded and frozen in cutting compound (Tissue-Tek O.C.T., Redding, Calif), then sectioned at $5 \mu \mathrm{m}$, and stained with hematoxylin and eosin.

\section{Patency Analysis}

SVG patency at 1 week was determined by blinded review of a computed tomographic angiogram (420-ms rotation, $100-150 \mathrm{~mL}$ contrast agent intravenously at $5 \mathrm{~mL} / \mathrm{s}$ ) with retrospective electrocardiographic gating. Patency was defined as any flow through the entire graft regardless of the presence of stenosis. The graft was classified as nonpatent if a stump was seen or if there 
TABLE 1. Potential risk factors for venous clot formation

\begin{tabular}{|c|c|c|c|c|}
\hline Characteristic & $\begin{array}{c}\text { VasoView unheparinized } \\
\text { control }(n=10)\end{array}$ & $\begin{array}{l}\text { VasoView preheparinized, } \\
\text { full dose }(n=10)\end{array}$ & $\begin{array}{l}\text { VasoView preheparinized, } \\
\text { low dose }(n=10)\end{array}$ & $\begin{array}{c}\text { ClearGlide } \\
\text { unheparinized }(n=10)\end{array}$ \\
\hline Male sex $(\%)$ & 80 & 80 & 80 & 80 \\
\hline Age (y) & $65.0 \pm 11.5$ & $64.9 \pm 10.6$ & $63.9 \pm 12.6$ & $63.4 \pm 8.8$ \\
\hline Body mass index & $30.1 \pm 7.7$ & $29.6 \pm 5.7$ & $27.9 \pm 4.7$ & $28.3 \pm 4.5$ \\
\hline Diabetes $(\%)$ & 20 & 40 & 50 & 40 \\
\hline Smoking history (\%) & 80 & 70 & 70 & 80 \\
\hline Preoperative heparin (\%) & 40 & 50 & 70 & 80 \\
\hline Preoperative clopidogrel (\%) & 20 & 10 & 0 & 10 \\
\hline Preoperative aspirin* (\%) & 80 & 80 & 90 & 80 \\
\hline Intraoperative aprotinin (\%) & 50 & 60 & 70 & 30 \\
\hline PVD $(\%)$ & 20 & 10 & 10 & 10 \\
\hline Preoperative TEG MA† & $65.6 \pm 6.4$ & $63.6 \pm 9.9$ & $67.1 \pm 7.7$ & $64.0 \pm 7.5$ \\
\hline
\end{tabular}

PVD, Peripheral vascular disease; TEG MA, maximum amplitude of the thromboelastography trace. $P=$ not significant for all comparisons (1-way analysis of variance and $\chi^{2}$ ). *Patients taking preoperative daily aspirin before hospital admission.

was no contrast in an area known by operative report to contain a graft, a validated definition of postoperative bypass graft failure. ${ }^{13}$

\section{Statistics}

The primary end point of this study was the difference in clot between groups as defined by the clot fraction and clot volume, analyzed with 1-way analysis of variance and the Bonferroni multiple comparison test. Patient and conduit characteristics were compared among all 4 groups by a 1-way analysis of variance or $\chi^{2}$ test for continuous and categorical variables, respectively. Correlations were sought between potential risk factors for clot formation and clot volume and clot fraction by regression analysis. To correct for the multiple comparisons that have been performed on our data set regarding SVG patency, ${ }^{14}$ we defined a $P$ value of $<$ .01 as significant when comparing patency rates. Statistical analysis was performed with the InStat statistical package (GraphPad Software, Inc, San Diego, Calif) with the assistance of a biostatistician (A.J.).

\section{Results}

\section{Study Characteristics}

Patient characteristics that might influence the tendency for venous thrombus formation were similar among the 4 patient groups (Table 1). The mean length of the SV tract that was harvested and imaged was $27.4 \pm 6.9 \mathrm{~cm}$, whereas the length used for the necessary graft(s) was $20.3 \pm 7.6 \mathrm{~cm}$ (74\% of total length used), with no significant differences in these lengths noted between the treatment groups. The average time required for intraoperative OCT scanning was $4.6 \pm 1.2$ minutes per SV. Mean clot volume and fraction were less in the grafted portion of SV $\left(7.1 \pm 20.1 \mathrm{~mm}^{3}\right.$ and $32 \% \pm 33 \%)$ than in the discarded portion $\left(9.5 \pm 17.1 \mathrm{~mm}^{3}\right.$ and $36 \% \pm 41 \%$ ), but these differences did not reach statistical significance.

\section{Intraluminal Clot}

The ability to diagnose intraluminal clot by OCT imaging was confirmed in registered histologic sections (Figure
2). Clot was detected by OCT in all 10 control SVs (ie, VasoView device without preheparinization), with $80 \%$ of these veins found to have clot strands throughout the majority of their lengths. Preheparinization before sealed $\mathrm{CO}_{2} \mathrm{EVH}$ (either low or high dose) yielded significant reductions in clot fraction (Figure 3) and clot volume (Figure 4). Additionally, significant reductions in clot fraction and volume were noted after EVH with an open $\mathrm{CO}_{2}$ insufflation system. When intraluminal clot was imaged within veins from the 3 intervention groups, it was normally of trivial volume compared with the clot observed in the control group. In the control group, both age $(r=0.66, P<.05)$ and preoperative TEG maximum amplitude $(r=0.97, P<.0001)$ were found to significantly correlate with intraluminal clot volume. None of the other risk factors for venous thrombus (listed in Table 1) were found to have an effect on intraluminal clot formation during $\mathrm{EVH}$.

Preheparinization was believed to increase the technical difficulty of left internal thoracic artery harvest and EVH, owing to increased oozing in the surgical field. However, intraoperative blood loss and perioperative and postoperative blood product requirements were similar in all patient groups. Intimal injury as visualized by OCT imaging was an unusual finding after EVH and almost always confined to the ostia of branch points. The incidence of these branch point tears was similar between groups (VasoView system $=13 \%$ vs ClearGlide system $=10 \%$ of SVs with at least one ostial branch tear; $P=$ not significant). Only one severe intimal tear was imaged within the procured SV, and that occurred after a VasoView harvest. The vast majority of clot strands (89\%) were observed to attach to the vessel wall at no discrete structure with only a minority attached to valves (6\%), branch points (4\%), and focal areas of trauma $(1 \%)$. 

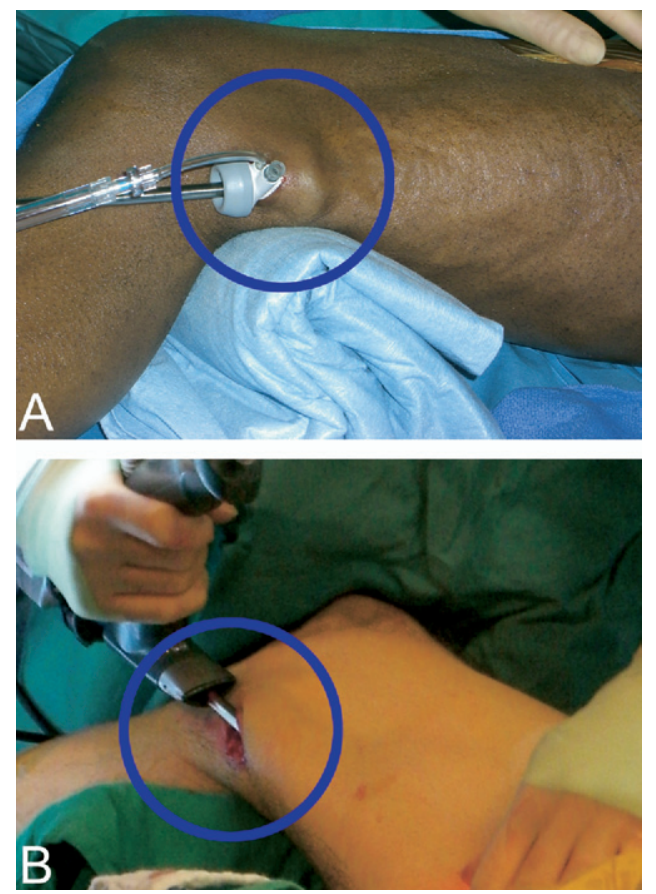

Figure 1. Sealed versus open $\mathrm{CO}_{2}$ insufflation EVH. The VasoView EVH system (Guidant Corp) (A) uses an inflated balloon to seal the incision site while $\mathrm{CO}_{2}$ is constantly insufflated to a pressure of 10 to $12 \mathrm{~mm} \mathrm{Hg}$ during harvest. In contrast, the ClearGlide EVH device (Datascope Corp) (B) does not rely on sealed $\mathrm{CO}_{2}$ insufflation. An open incision allows gas to freely escape from the harvesting tunnel. Although this modification may make visualization more challenging with the ClearGlide device, it allows for more consistent blood flow within the SV during harvest and approximates an open harvesting method in this regard.

\section{SVG Patency}

Four SVGs in the control group were occluded (patency rate $=69 \%$ ) at 1 week, whereas only 1 SVG was occluded in the 3 intervention groups (patency rate $=97 \%$ ), but these differences did not reach statistical significance. The mean clot volume and fraction in occluded SVGs was $29.7 \pm 38.1$ $\mathrm{mm}^{3}$ and $0.57 \pm 0.34$, as compared with $6.3 \pm 15.7 \mathrm{~mm}^{3}$ and $0.26 \pm 0.31$ in patent grafts $(P=$ not significant $)$.

\section{Discussion}

Clot strands after EVH have been previously described. ${ }^{8}$ However, without a practical method for intraoperative imaging of conduits, the actual volume and distribution of clot retained within the SV lumen after flushing has never been fully appreciated. In this study, we establish that OCT is able to accurately identify the incidence and severity of this problem, as well as provide the necessary sensitivity for detecting small changes in the volumes of clot after alterations in standard EVH practice. Because this OCT examination can be done in real time, intraoperatively, and with minimal interference in the normal surgical protocol (average scan time $=4.6$ minutes), this method represents an important quality assurance tool for investigating the effectiveness of various conduit harvest protocols. We noted that clot retained within the SV was a universal and specific finding to $\mathrm{EVH}$ with sealed $\mathrm{CO}_{2}$ insufflation in the absence of heparinization before initiation of the procedure. Use of open $\mathrm{CO}_{2}$ insufflation to maintain flow within the $\mathrm{SV}$, or systemic administration of heparin before occluding flow using a sealed $\mathrm{CO}_{2}$ system, both led to dramatic reductions in this retained clot. These findings support our hypothesis that clot formation during EVH is the result of stagnant blood that is not anticoagulated being allowed to remain within a collapsed SV.

In assessing preoperative risk factors for clot formation in the control group, we found both age and the maximum amplitude of the TEG trace (a measure of platelet function) to be significantly correlated with intraluminal clot volume. Our data corroborate previous reports suggesting that the risk for hypercoagulability is linked to increased age ${ }^{15}$ and that TEG maximum amplitude specifically correlates with the risk of postoperative deep vein thrombosis. ${ }^{16}$ Although
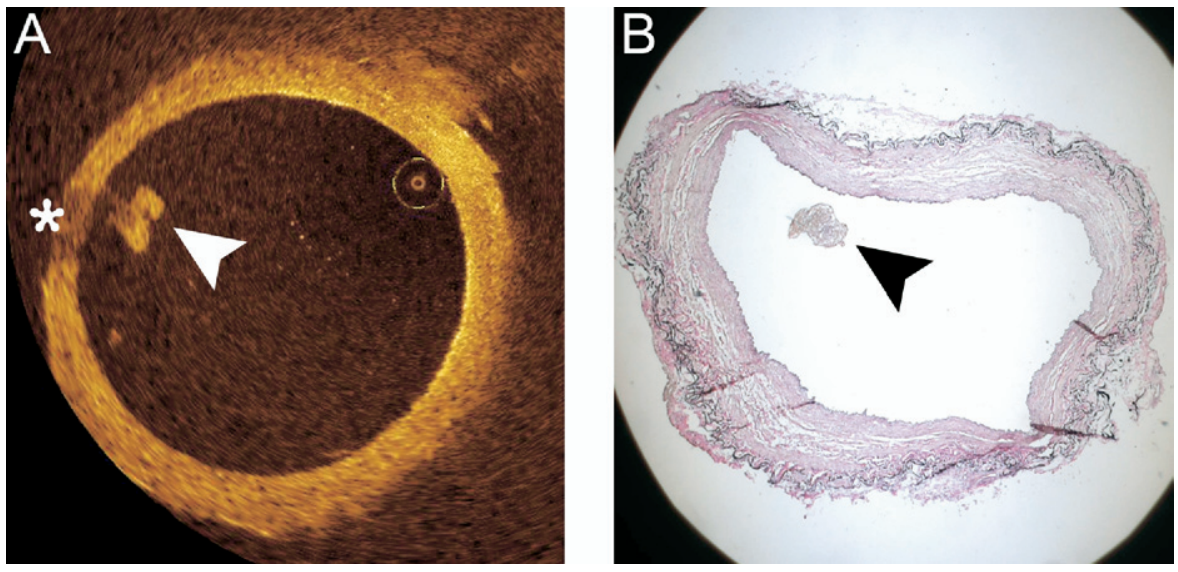

Figure 2. Histologic correlation of intraluminal clot. Intraluminal clot (arrowheads) is visible as a signal-rich lobulated mass via OCT (A) and in a registered histologic section (B). Note the characteristic radial signal attenuation that the clot produces on OCT imaging (asterisk). 


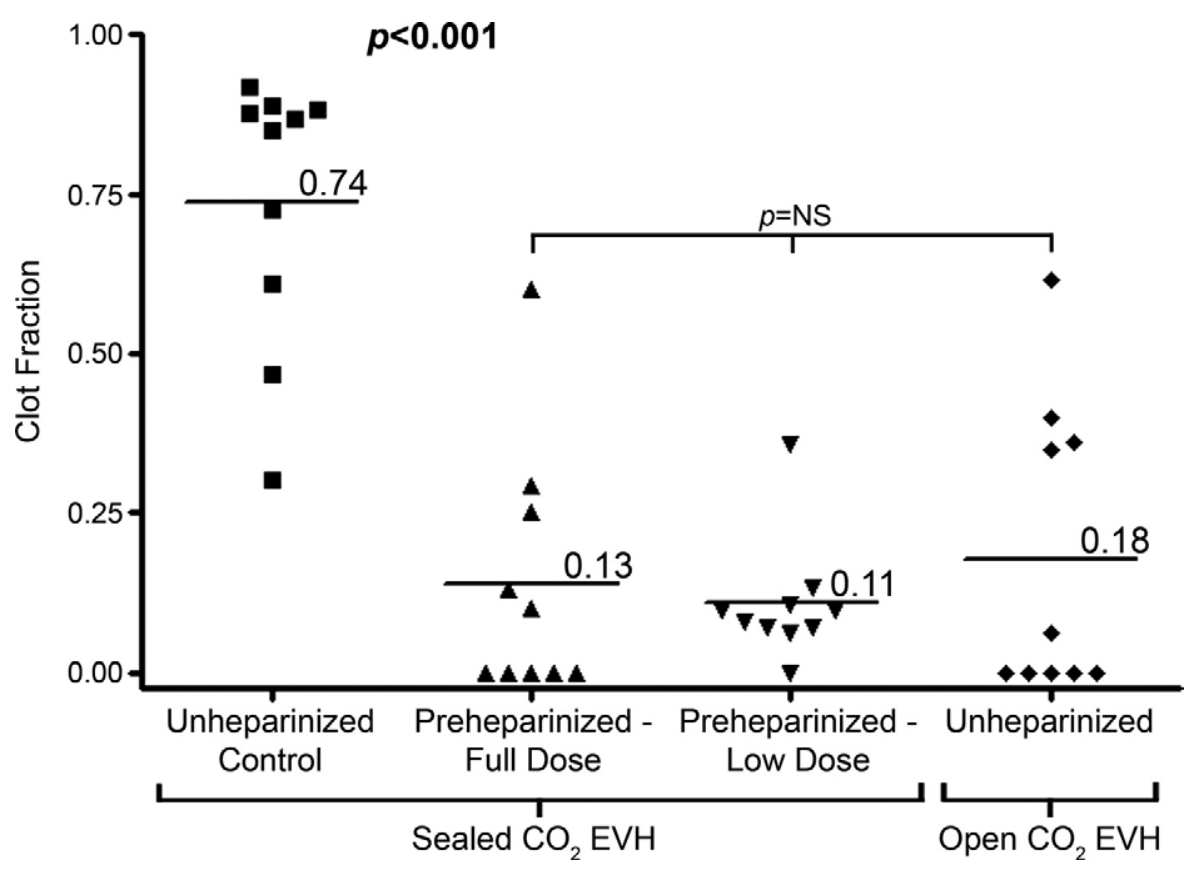

Figure 3. Clot fraction. Intraluminal clot was a universal finding in unheparinized control veins with $80 \%$ containing clot throughout the majority of their length. Preheparinziation, using both full (titrated to activated clotting time $>300$ seconds) and low (5000-IU bolus) doses, was associated with a significant reduction in overall fraction of the vein containing clot, with many veins containing no clot at all. Open $\mathrm{CO}_{2} \mathrm{EVH}$ yielded a similar reduction in clot fraction without preheparinization.

these data suggest that elderly patients with elevated TEG maximum amplitude are the most likely to benefit from the interventions described in this report, the small number of patients included in this analysis places us at risk for a type I statistical error. Clarification of which patients are at greatest risk from SV harvest via the VasoView device without preheparinization awaits further study.
Preheparinization did not change blood loss or transfusion requirements, suggesting that it is a benign and effective method for avoiding clot strands when the VasoView system is used. However, EVH with open $\mathrm{CO}_{2}$ insufflation significantly reduced the volume and length of retained clot within the conduit without the need for anticoagulation before the completion of sternotomy and left internal tho-

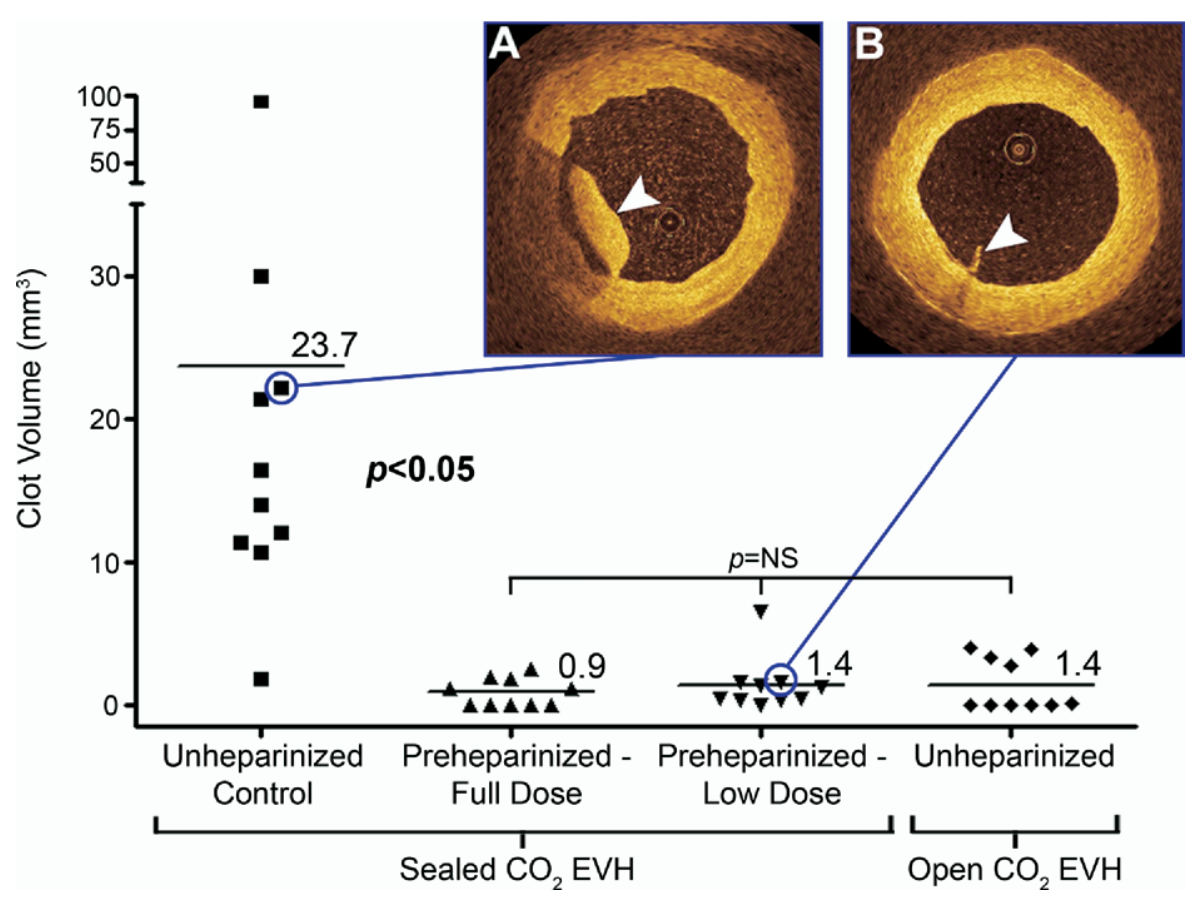

Figure 4. Clot volume. Preheparinziation, using both full (titrated to activated clotting time $>300$ seconds) and low (5000-IU bolus) doses, was associated with a significant decrease in clot volume. Open $\mathrm{CO}_{2} \mathrm{EVH}$ without preheparinization produced a similar reduction. When clot was present in preheparinized or open $\mathrm{CO}_{2}$ EVH veins, it was normally of trivial volume (B, arrowhead) compared with the substantial volumes imaged in the unheparinized control group (A, arrowhead). 
racic artery takedown. The benefit of this alternative harvesting system is likely derived by maintaining constant blood flow within the SV during EVH. It is important to note that our group and others ${ }^{17}$ have found endoscopic visualization to be more technically challenging with an open $\mathrm{CO}_{2}$ insufflation system.

Our study was not appropriately powered to determine whether intraluminal clot strands predispose to graft failure. A recent study showed that 5-year event-free survival (freedom from death, myocardial infarction, or recurrent angina) was not significantly different in patients with SVGs procured via EVH versus open harvest. ${ }^{18}$ However, this study used an open $\mathrm{CO}_{2} \mathrm{EVH}$ system and, as evidenced by our findings, cannot be extrapolated to EVH with the VasoView device. A meta-analysis based on several small randomized trials suggested no adverse effect of EVH on angiographic patency. ${ }^{4}$ Again, of the 3 trials that comprised the data in this report, only the trial by Perrault and associates ${ }^{19}$ used a closed $\mathrm{CO}_{2}$ insufflation EVH system without preheparinization. This trial included only 40 patients and showed a nonsignificant trend toward reduced patency of SVGs harvested with a protocol that, as we now know, is prone to intraluminal clot formation.

In contrast, the recent PREVENT IV multicenter trial ${ }^{20}$ was appropriately powered to assess graft patency and used predominantly closed $\mathrm{CO}_{2}$ insufflation during EVH. Although not dictated by the protocol or specifically described in the report, it can be assumed that the timing of heparin administration followed the common practice at most centers, which is to avoid heparinization until after internal thoracic artery (and therefore SV) harvest. In this large report, the use of EVH instead of an open technique was an independent predictor of graft failure. ${ }^{5}$ Endothelial integrity has been found to be similar by our group and others ${ }^{2,6,7}$ between SVs harvested by these 2 techniques. Taken together, these data strongly suggest that intraluminal clot strands that are retained after endoscopic procurement may have played a causal role in the higher risk of SVG loss after EVH observed in the PREVENT IV study.

Our study has limitations. Group assignment was not randomized, which increases the risk that bias may have influenced our results. We believe that the impact of any bias introduced by this study design was minimized because patient characteristics that would be likely to affect clot formation were found to be similar between groups and SV clot was analyzed by a single technician blinded to group assignment. Our study was statistically powered to demonstrate the effects of changes to the EVH protocol on clot formation but not to address the mechanism or pathogenicity of clot formation or the risks of preheparinization. The results of this study are most appropriately used as preliminary data to support the design of a randomized trial that will provide more definitive conclusions on these issues.
By enabling the quantification of this issue as never before possible, OCT screening revealed that retained clots are frequently found within the SV after EVH. Our pilot data suggest that heparinization before EVH or use of an open $\mathrm{CO}_{2}$ system are benign changes in practice that can significantly improve this complication. Further studies are required to determine the pathogenicity of this finding.

\section{References}

1. Puskas JD, Wright CE, Miller PK, Anderson TE, Gott JP, Brown WM, et al. A randomized trial of endoscopic versus open saphenous vein harvest in coronary bypass surgery. Ann Thorac Surg: 1999;68:1509-12.

2. Kiaii B, Moon BC, Massel D, Langlois Y, Austin TW, Willoughby A, et al. A prospective randomized trial of endoscopic versus conventional harvesting of the saphenous vein in coronary artery bypass surgery. J Thorac Cardiovasc Surg: 2002;123:204-12.

3. Bonde P, Graham AN, MacGowan SW. Endoscopic vein harvest: advantages and limitations. Ann Thorac Surg: 2004;77:2076-82.

4. Cheng D, Allen K, Cohn W, Connolly M, Edgerton J, Falk V, et al. Endoscopic vascular harvest in coronary artery bypass grafting surgery: a meta-analysis of randomized trials and controlled trials. Innovations Technol Tech Cardiothorac Vasc Surg. 2005;1:61-74.

5. Magee MJ, Alexander JH, Hafley G, Ferguson TB, Gibson CM, Harrington RA, et al. Saphenous vein graft failure and clinical outcomes following on-pump and off-pump coronary artery bypass: findings from Prevent IV. Presented at the 53rd Annual Meeting of the Southern Thoracic Surgical Association, Tucson, AZ, 2006.

6. Griffith GL, Allen KB, Waller BF, Heimansohn DA, Robison RJ, Schier JJ, et al. Endoscopic and traditional saphenous vein harvest: a histologic comparison. Ann Thorac Surg. 2000;69:520-3.

7. Manchio JV, Gu J, Romar L, Brown J, Gammie J, Pierson RN, et al. Disruption of graft endothelium correlates with early failure after off-pump coronary artery bypass surgery. Ann Thorac Surg. 2005;79: 1991-8.

8. Burris NS, Schwartz K, Brown JM, Kwon M, Pierson RN, Griffith BP, et al. Incidence of residual clot strands in saphenous vein grafts after endoscopic harvest. Innovations Technol Tech Cardiothorac Vasc Surg. 2006;1:323-7.

9. Burris NS, Schwartz K, Tang CM, Jafri MS, Schmitt J, Kwon MH, et al. Catheter-based infrared light scanner as a tool to assess conduit quality in coronary artery bypass surgery. J Thorac Cardiovasc Surg.. 2007;133:419-27.

10. He GW, Rosenfeldt FL, Angus JA. Pharmacological relaxation of the saphenous vein during harvesting for coronary artery bypass grafting. Ann Thorac Surg. 1993;55:1210-7.

11. Poston RS, White C, Gu J, Brown J, Gammie J, Pierson RN, et al. Aprotinin shows both hemostatic and antithrombotic effects during off-pump coronary artery bypass grafting. Ann Thorac Surg.. 2006; 81:104-10.

12. Kume T, Akasaka T, Kawamoto T, Ogasawara Y, Watanabe N Toyota E, et al. Assessment of coronary arterial thrombus by optical coherence tomography. Am J Cardiol. 2006;97:1713-7.

13. Frazier AA, Qureshi F, Read KM, Gilkeson RC, Poston RS, White CS. Coronary artery bypass grafts: assessment with multidetector CT in the early and late postoperative settings. Radiographics. 2005;25:881-96.

14. Poston RS, Gu J, Brown JM, Gammie JS, White C, Nie L, et al. Endothelial injury and acquired aspirin resistance as promoters of regional thrombin formation and early vein graft failure after coronary artery bypass grafting. J Thorac Cardiovasc Surg. 2006;131:122-30.

15. Abbate R, Prisco D, Rostagno C, Boddi M, Gensini GF. Age-related changes in the hemostatic system. Int J Clin Lab Res. 1993;23:1-3.

16. Sugiura K, Ikeda Y, Ono F, Watanabe K, Ando Y. Detection of hypercoagulability by the measurement of the dynamic loss modulus of clotting blood. Thromb Res. 1982;27:161-6.

17. Dalrymple-Hay MJ, Alzetani A, Costa R, Ohri SK. Endoscopic vein harvesting with the aid of carbon dioxide insufflation. Ann Thorac Surg. 2001;71:739-41. 
18. Allen KB, Heimansohn DA, Robison RJ, Schier JJ, Griffith GL, Fitzgerald EB. Influence of endoscopic versus traditional saphenectomy on event-free survival: five-year follow-up of a prospective randomized trial. Heart Surg Forum. 2003;6:E143-5.

19. Perrault LP, Jeanmart H, Bilodeau L, Lespérance J, Tanguay JF, Bouchard D, et al. Early quantitative coronary angiography of saphenous vein grafts for coronary artery bypass grafting harvested by means of open versus endoscopic saphenectomy: a prospective randomized trial. J Thorac Cardiovasc Surg. 2004;127:1402-7.

20. Alexander JH, Hafley G, Harrington RA, Peterson ED, Ferguson TB, Lorenz TJ, et al. Efficacy and safety of edifoligide, an E2F transcription factor decoy, for prevention of vein graft failure following coronary artery bypass graft surgery: PREVENT IV-a randomized controlled trial. JAMA. 2005;294:2446-54.

\section{Discussion}

Dr Michael Mack (Dallas, Tex). A paper presented at the Southern Thoracic Surgical Association meeting last year analyzed the PREVENT IV trial for graft patency and found that endoscopic vein harvest was a risk factor for lower graft patency. I think there is some evidence that this may have some clinical impact, and you may have defined the mechanism.

It is always said to be dangerous to go home after a medical meeting because people change their practices based on what they heard at the meeting. On the basis of your presentation, I think I would seriously consider earlier heparinization in patients. Have you seen any adverse clinical sequelae from earlier heparinization, such as late complications or hematoma of the harvest tunnel?

Dr Brown. We have not seen any complications such as those. Increased bleeding is an obvious concern with preheparinization and seems to be one of the reasons that people are reluctant to use it. However, we found no effect of preheparinization on intraoperative blood loss volume or perioperative and postoperative transfusion requirements. The only potential complication we noted was that the surgical field was not quite as dry. There was some increased oozing of blood during harvest of the internal thoracic artery.

Dr Valavanur Subramanian (New York, NY). I have two questions. Did you determine the age of the clot or the type of the clot in the SVGs? That would be very important for us.

Dr Brown. We are currently trying to assess the morphology of this intraluminal clot. One intervention that we have also tried is using tissue plasminogen activator to lyse this clot, but this strategy has not proved very effective at decreasing clot volume. We have previously documented that this intraluminal clot contains platelets, which may be contributing to the failure of lysis we are observing. Therefore, we believe our tissue plasminogen activator incubation solution might benefit from the incorporation of a glycoprotein Ilb/IIIa inhibitor. Obviously, determining the exact morphology of this intraluminal thrombus would be important in this effort.
Dr Subramanian. The second question concerns infusion of heparin through the cut end of the vein graft before you do the EVH. Would that be helpful rather than 5000 units?

Dr Brown. That is something we have also considered. The problem we encountered with the strategy you describe was that the vein harvester did not want to be committed to using that particular section of the vein. However, some other people have also suggested that technique, and it really just depends on whether the surgeon is comfortable doing that.

Dr A. W. Atkinson (Raleigh, NC). I think it is a wonderful study. Interestingly, in our community we have two hospitals and we have two different techniques for harvesting vein: one is an open system, I believe the Ethicon system, and the other is a closed system with $\mathrm{CO}_{2}$ pressure. When we go from one hospital to the other, we obviously see more overt and after we harvest the vein we see more interluminal thrombus. We have actually started giving preharvest heparin in small doses. It is not a scientific study at all, but observationally we saw a marked decrease in interluminal thrombus. We are doing the study in a sense just from one hospital to the other. I really commend you on documenting this observation that we have seen.

I had the same thought as Dr Subramanian. Why don't we just make a small incision at the ankle and flush it with heparinized saline before harvesting to obviate both problems? I think we may start to do that until we get it further documented. However, it is a wonderful study with a new technology that we should look at with other aspects of vein harvesting.

Do you have any way to identify vein trauma with this technology or some other technology about intimal damage? It definitely occurs differently with different techniques.

Dr Brown. Thank you. I realize other surgeons have already begun giving heparin before $\mathrm{EVH}$, and I think one of the exciting things about OCT is that it has now allowed us to truly quantitate the efficacy of this strategy for reducing intraluminal clot burden.

OCT is also very sensitive for detecting intimal damage. It obviously has much higher resolution than intravascular ultrasound, and we are currently using it to determine conduit quality intraoperatively, especially in regard to harvesting-related trauma. Here you can see an intimal tear, and we normally see this occur just around branch points. We also use OCT to evaluate radial arteries, and in those vessels we see some more overt trauma than in the SV, probably owing to the more friable nature of the radial.

Dr Atkinson. Do you have further studies planned comparing harvesting techniques with intimal damage?

Dr Brown. We are conducting studies like this with the radial artery. However, it should be noted that we do not see harvestingrelated trauma very often in the $\mathrm{SV}$ after $\mathrm{EVH}$, so our current trials are assessing only the radial artery. 